\title{
Effect of bamboo (Phyllostachys pubescens) extract on broiler chickens under cold stress
}

\author{
Xingyong Chen ${ }^{1}$, Runshen Jiang ${ }^{1}$, Xuede $\mathrm{Li}^{2}$, Yongde Yue ${ }^{3}$, Zhaoyu Geng ${ }^{1 \#}$ \\ ${ }^{1}$ College of Animal Science and Technology, Anhui Agricultural University, Hefei 230036 China \\ ${ }^{2}$ Resources and Environment College, Anhui Agricultural University, Hefei 230036 China \\ ${ }^{3}$ International Bamboo \& Rattan Centre, Beijing 100102, China
}
Copyright resides with the authors in terms of the Creative Commons Attribution 2.5 South African Licence.
See: http://creativecommons.org/licenses/by/2.5/za/
Condition of use: The user may copy, distribute, transmit and adapt the work, but must recognise the authors and the South African Journal of Animal Science

\begin{abstract}
The objective of the study was to establish whether powdered bamboo extract (BFRE) as a feed additive for chickens was effective in maintaining body weight gain and serum enzymatic activity when birds are exposed to cold stress. Broiler Huainan partridge chickens (4 weeks old), fed a diet containing powdered BFRE $(0.3 \mathrm{~g} / \mathrm{kg})$ for one week, showed significant weight gains compared to the control. Subsequently, the chickens were exposed to low temperatures $\left(15\right.$ or $\left.10{ }^{\circ} \mathrm{C}\right)$ for one week with or without BFRE addition. After one week of exposure to 15 or $10^{\circ} \mathrm{C}$, serum enzymatic activities were recorded. Chickens not receiving BFRE and exposed to $10{ }^{\circ} \mathrm{C}$ showed a significant decrease in the body weight gain and in serum creatine kinase, lactic dehydrogenase $\mathrm{L}$ and alanine aminotransferase activities, whereas the activity of these enzymes changed to a lesser extent in chickens receiving BFRE. However, body weight gains were maintained within the normal range when the ambient temperature was $10^{\circ} \mathrm{C}$. In conclusion, BFRE was an effective additive in preventing chickens from losing body weight during cold stress, and in fluctuations in serum enzymatic activity when exposed to cold stress. The results suggest that powdered BFRE is an effective additive in minimizing the negative effects of cold stress in broiler chickens.
\end{abstract}

Keywords: Huainan partridge chicken, powdered bamboo extract, weight gain, serum enzymatic activity

\# Corresponding author: xy9chen@ mail.ustc.edu.cn

\section{Introduction}

Broiler chickens reared at low temperatures encompasses a challenge to producers (Shinder et al., 2007). Chickens are highly sensitive to environmental temperatures which should be controlled within an appropriate range for optimal performance. A standard rearing practice is to maintain the environmental temperature at which neonatal chickens are reared at about $32{ }^{\circ} \mathrm{C}$ for about four weeks and then to decrease it gradually. However, without external heating, a sudden decrease of environmental temperature during the neonatal stage can have a negative impact on chickens, such as a higher susceptibility to diseases and a decrease in body weight gain. This in turn affects production performance (Yahav, 2000). The Anhui Province is located in southeast China with an average temperature of $12.3{ }^{\circ} \mathrm{C}$ in winter. Integrated management and wind insulation devices have been adopted in chicken houses during rearing to maintain an environmental temperature of between 12 and $16{ }^{\circ} \mathrm{C}$. Therefore, it is imperative to determine whether feed additives could assist in maintaining the growth rate of broiler chickens when fluctuations in temperature occur at these low temperatures without external heating.

Bamboo (Phyllostachys pubescens) grows particularly well in south-eastern Asia and represents a traditionally important commodity that is used for building material, in traditional medicine and as a source of food. The bioactivity of bamboo extract has been studied widely (Chuyen et al., 1982). Flavonoids, bioactive polysaccharides, phenolic acids, anthraquinone, coumarin lactone, special amino acids, aromatic components and several trace elements have been identified in bamboo extracts and have been evaluated in vitro for free radical scavenging and antioxidant activities (Hu et al., 2000). Studies have reported that the supplementation of powdered wood vinegar compounds and amorphous charcoal carbon elicited positive responses in animal production (Samanya \& Yamauchi, 2001; Mekbungwan et al., 2007). The addition of 
wood vinegar compounds to diets induced significant increases in hen-day egg production and feed conversion ratio, and in broiler hatchability. Bamboo extract, with components similar to powdered wood vinegar compounds, is known for its anti-microbial effects (Wang \& Ng, 2003), but few investigations have been conducted to assess its efficacy as a feed additive in poultry during cool seasons.

In this study it was assessed whether powdered bamboo extract (BFRE) as a chicken feed additive could assist in protecting birds exposed to cold stress to maintain body weight gain and serum enzymatic activity in chickens.

\section{Materials and Methods}

Bamboo extract was supplied by the Resources and Environment College of the Anhui Agricultural University, China. The extraction process was as follows: a total of $2.1 \mathrm{~kg}$ ground bamboo powder was sieved through a 40-mesh into a container and 95\% ethanol was added (bamboo powder : ethanol = 1:5). It was sealed and stored for seven days. The paste extract was collected using a Büchner funnel and then concentrated by drying at $55^{\circ} \mathrm{C}$ with a rotary evaporator (Cao et al., 2005).

The broilers used were an indigenous Chinese breed, the Huainan partridge chicken. Four hundred and fifty physically healthy males were obtained from the Feixi the Old Hens Farming Co., LTD at four weeks of age. Body weights were measured weekly between 8:00 - 10:00 using a digital balance (ACS-30, Dayang, China). Chickens weighing between 210 - $230 \mathrm{~g}$ were randomly divided into five groups (90 per group). For the first week the chickens were housed at a constant environmental temperature of $20 \pm 2{ }^{\circ} \mathrm{C}$ with ad libitum access to water and a commercial diet (metabolizable energy: $11.66 \mathrm{MJ} / \mathrm{kg}$, crude protein: $195 \mathrm{~g} / \mathrm{kg}$ ). In the second week $0.03 \%$ BFRE was added to the diets of groups 4 and 5 and in the third week group 1 (control) was kept at $20 \pm 2{ }^{\circ} \mathrm{C}$. Groups 2 and 4 were exposed to $15 \pm 2{ }^{\circ} \mathrm{C}$, with group 4 receiving the experimental diet containing BFRE, and groups 3 and 5 were exposed to $10 \pm 2{ }^{\circ} \mathrm{C}$, with group 5 receiving the diet containing BFRE (Table 1).

Table 1 Grouping and management of Huainan partridge chickens

\begin{tabular}{|c|c|c|c|c|c|}
\hline Age (weeks) & Group one & Group two & Group three & Group four & Group five \\
\hline 4 & \multicolumn{5}{|c|}{$20 \pm 2{ }^{\circ} \mathrm{C}$ on Commercial chicken diet } \\
\hline 5 & \multicolumn{3}{|c|}{$20 \pm 2{ }^{\circ} \mathrm{C}$ on Commercial chicken diet } & \multicolumn{2}{|c|}{$\begin{array}{c}20 \pm 2{ }^{\circ} \mathrm{C} \text { on Commercial chicken } \\
\text { diet with } 0.03 \% \text { BFRE }\end{array}$} \\
\hline \multirow[b]{2}{*}{6} & $20 \pm 2{ }^{\circ} \mathrm{C}$ & $15 \pm 2{ }^{\circ} \mathrm{C}$ & $10 \pm 2{ }^{\circ} \mathrm{C}$ & $15 \pm 2{ }^{\circ} \mathrm{C}$ & $10 \pm 2{ }^{\circ} \mathrm{C}$ \\
\hline & \multicolumn{3}{|c|}{ Commercial chicken diet } & \multicolumn{2}{|c|}{$\begin{array}{l}\text { Commercial chicken diet } \\
\text { with } 0.03 \% \text { BFRE }\end{array}$} \\
\hline
\end{tabular}

Note 1: Chickens at 4 - 5 week age in Group one to five all housed at $20 \pm 2{ }^{\circ} \mathrm{C}$. In $5 \mathrm{~W}$, Groups one to three received the commercial diet and Groups four and five the diet containing 0.03\% BFRE. During week 6, Group one was housed at $20 \pm 2{ }^{\circ} \mathrm{C}$, Group two at $15 \pm 2{ }^{\circ} \mathrm{C}$ and Group 3 at $10 \pm 2{ }^{\circ}$, all receiving the experimental diet. Group four was housed at $15 \pm 2{ }^{\circ} \mathrm{C}$ and Group five at $10 \pm 2{ }^{\circ} \mathrm{C}$. Both these groups receiving the experimental diet with $0.03 \%$ bamboo extract (BFRE) added.

After the second week, following a $12 \mathrm{~h}$ fasting, a $2 \mathrm{~mL}$ sample of blood was collected from the jugular vein from 30 chickens in each group, using sterilized syringes. All efforts were made to minimize pain or discomfort of the chicks. The blood samples were centrifuged at $3500 \mathrm{rpm}$ for $10 \mathrm{~min}$ at $4{ }^{\circ} \mathrm{C}$ after 20 min in a water bath at $37{ }^{\circ} \mathrm{C}$ to collect serum. None of the samples was haemolyzed.

Alanine aminotransferase (ALT, EC 2.6.1.2), aspartate aminotransferase (AST, EC 2.6.1.1) and gamma-glutamyltransferase $(\gamma$-GT, EC 2.3.2.2) activities were measured by kinetic assay, and creatine kinase (CK, EC 2.7.3.2) activity by enzyme coupling spectrophotometry (Sun et al., 2007). The activity of lactate dehydrogenase L (LDH-L, EC 1.1.1.27) was measured using a lactic kinetic assay, and triiodothyronine (T3), thyroxine (T4), thyroid-stimulating hormone (TSH) and insulin concentrations were 
determined using the ROCHE E2010/ABBOTT I2000 automatic biochemical analyzer and related testing kits (Hangalapura et al., 2004).

Data were analysed using the Proc GLM procedure of SAS (SAS 9.1, 2001). The data on each serum enzymes and biochemical measurement were grouped and expressed as mean pooled standard errors of the mean. Data (mean \pm SEM) were considered to be statistically significant when the value of $P$ was less than 0.05 or 0.01 . The statistical model used was: $\mathrm{Yij}=\mu+\alpha \mathrm{i}+\mathrm{Eij}$ : Where $\mathrm{Yij}=$ body weight change, serum enzyme or insulin levels; $\mu=\mathrm{a}$ constant; $\alpha \mathrm{i}=$ effect of feed method (Groups 1 to 5); Eij = random error; with the assumption that data were normally distributed with equal variances.

\section{Results}

The body weight gains of the Huainan partridge chickens were measured after differential temperature exposure with or without $0.03 \%$ BFRE addition (Table 2). During the first experimental week, chickens were kept under the same environmental conditions. After one week of $0.03 \%$ BFRE addition, the body weights of chickens in Groups four and five were higher $(P<0.05)$ than those of the non-BFRE addition group (Group one to three). In the week that followed, chickens were exposed to different environmental temperatures. The BFRE addition groups had nearly the body same weight as the control group. However, chickens in the nonBFRE addition group exposed to $10{ }^{\circ} \mathrm{C}$ experienced a decreased body weight gain $(P<0.05)$, while those exposed to $15{ }^{\circ} \mathrm{C}$ showed no obvious effect except for a decreasing trend in body weight gain.

After one week of hypothermia exposure $\left(15^{\circ} \mathrm{C}\right.$ or $\left.10^{\circ} \mathrm{C}\right)$ serum from each group was collected and the activities of the serum enzymes, ALT/GPT, $\gamma$-GT, CK, LDH-L and AST, as well as concentrations of the serum biochemical indices, T3, T4, TSH and insulin, were measured (Figures 1 and 2).

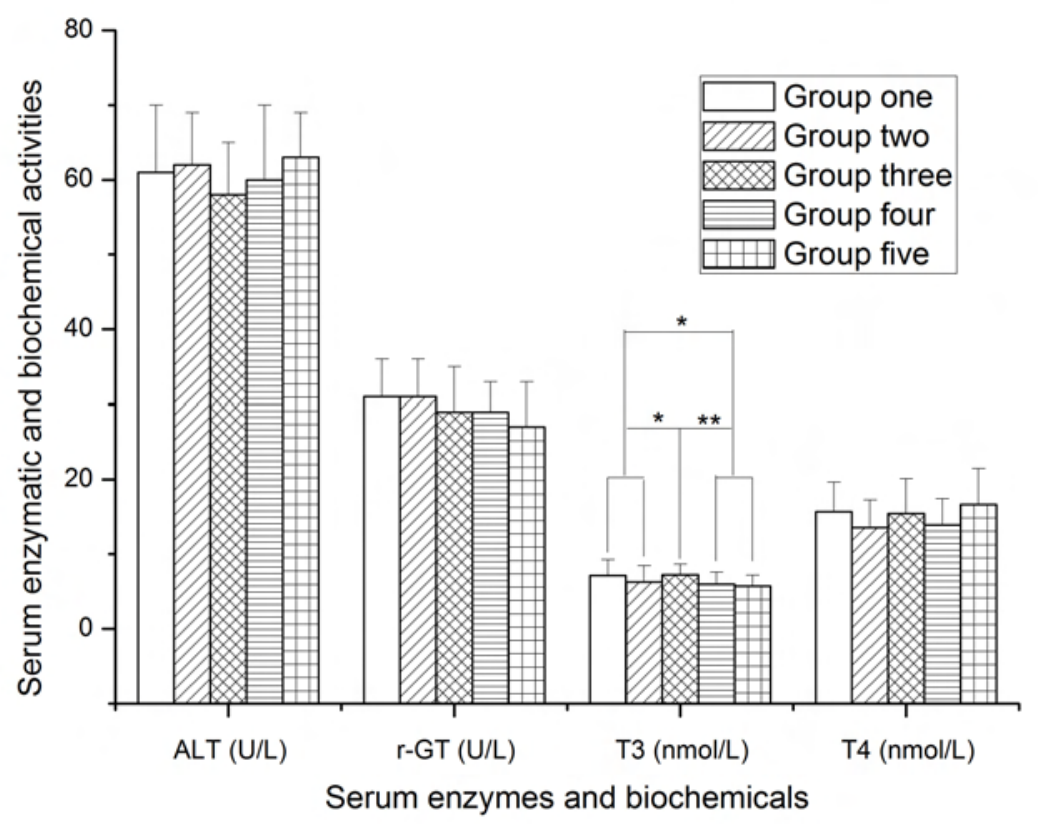

Figure 1 Serum gamma-glutamyltransferase $(\gamma$-GT) and alanine aminotransferase (ALT) activities, and triiodothyronine (T3) and thyroxine (T4) concentrations in Huainan partridge chickens at different temperatures and additive. Values are means \pm SD. $* P<0.05$, $* * P<0.01$.

One week hypothermia exposure of $15^{\circ} \mathrm{C}$ induced a decrease $(P<0.05)$ in CK, LDH-L and AST activity. When the ambient temperature was $10{ }^{\circ} \mathrm{C}$, significant decreases $(P<0.01)$ in CK, LDH-L and AST activities were recorded. Serum indices were also tested for chickens fed the $0.03 \%$ BFRE and exposed to 
the same environmental temperatures $\left(15^{\circ} \mathrm{C}\right.$ or $\left.10{ }^{\circ} \mathrm{C}\right)$. The results indicated that serum indices were similar to those in the control group with. The ALT and $\gamma$-GT activities did not differ between the groups receiving no BFRE and those receiving BFRE in their diets. Considering the thyroid related indicators and insulin, the results showed an elevation $(P<0.05)$ in group 3 (exposed to $\left.10{ }^{\circ} \mathrm{C}\right)$ and a decrease $(P<0.05)$ in groups 4 and 5 (receiving BFRE). No differences in T4, TSH and insulin concentrations (data not shown) were recorded between treatments.

Table 2 Body weight (g) changes of Huainan partridge chickens in each experimental group at six weeks of age

\begin{tabular}{cccccc}
\hline Week age & Group one & Group two & Group three & Group four & Group five \\
\hline $4 \mathrm{~W}$ & & & $220.3 \pm 20.1$ & & \\
$5 \mathrm{~W}$ & $286.8^{\mathrm{A}} \pm 32.3$ & $285.0^{\mathrm{A}} \pm 36.4$ & $284.9^{\mathrm{A}} \pm 31.1$ & $308.3 \pm 34.4^{\mathrm{B}}$ & $311.2^{\mathrm{B}} \pm 35.1$ \\
$6 \mathrm{~W}$ & $368.7^{\mathrm{A}} \pm 47.3$ & $361.8^{\mathrm{AB}} \pm 47.8$ & $346.2^{\mathrm{B}} \pm 51.6$ & $369.9^{\mathrm{A}} \pm 48.2$ & $369.1^{\mathrm{A}} \pm 47.7$
\end{tabular}

Body weight was compared in each group at the same week age.

${ }^{\mathrm{A}, \mathrm{B}}$ Different superscripts within rows indicate significant differences at $P<0.05$.

These results suggest that at a moderate decrease in temperature (group two), serum enzymatic and biochemical indices show no changes while a severe temperature decrease (group 3) caused significant fluctuations. However, with a $0.03 \%$ BFRE addition (groups four and five), there was no significant change in the experimental groups and minor change in the control group compared with groups two and three.

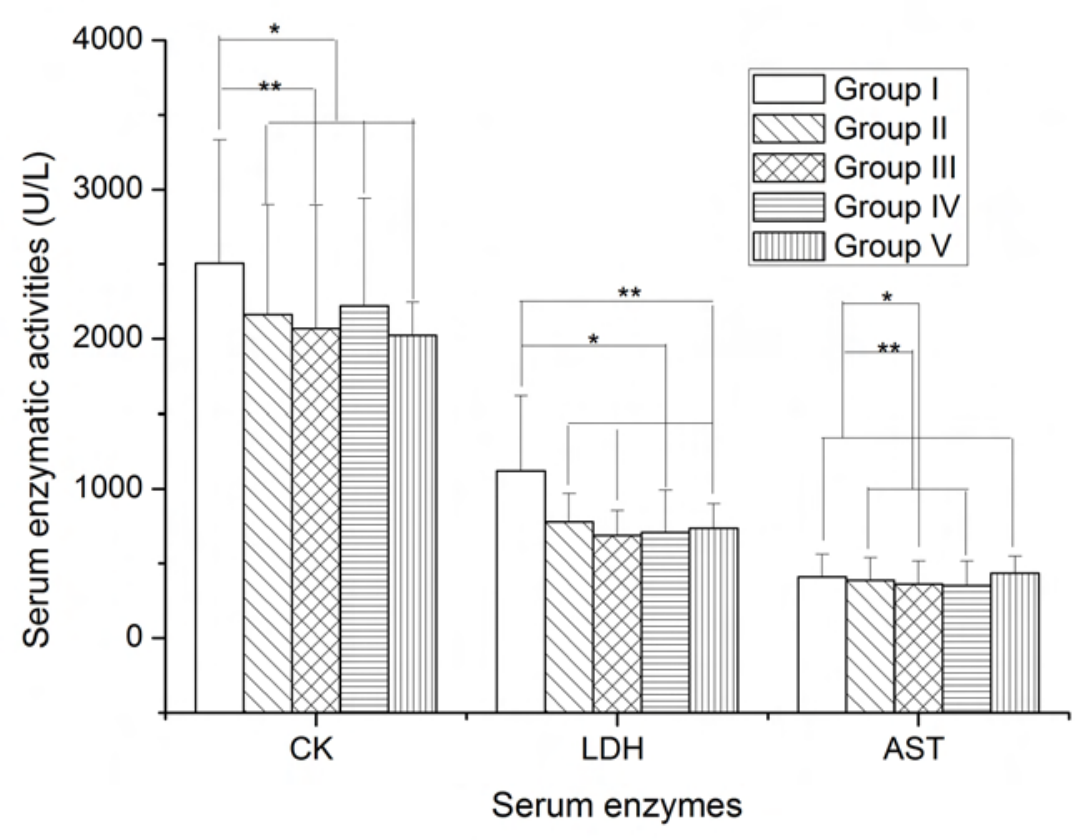

Figure 2 Serum creatine kinase (CK), lactate dehydrogenase (LDH-L) and aspartate aminotransferase (AST) activity in Huainan partridge chickens subjected to different temperatures, with or without bamboo extract (BFRE).

Values are means \pm SD. $* P<0.05, * * P<0.01$. 


\section{Discussion}

Several investigations have demonstrated that stress can induce changes in a variety of indices in chickens (Mujahid et al., 2007; Feng et al., 2008; Mujahid et al., 2009; Yan et al., 2009). Plant extracts always showed immuno-modulatory (Fakeye, 2008), anti-inflammatory (Castaldo \& Capasso, 2002), antimicrobial and growth promoting effects (Kumari et al., 2007). It has been reported that plant extracts often contain flavonoids, bioactive polysaccharides, and other that have been found to be beneficial in promoting chicken growth and resistance to disease. However, there is no report to date considering the alleviation by plant extracts of cold stress-induced damage in chickens. The BFRE is known for its anti-microbial usage. Findings from the present experiment indicated that BFRE might be a prospective additive to alleviate the effects of cold stress in poultry.

The results of the present study have shown that body weight gain was slow and most of the plasma parameters were found to be affected by cold stress but remained within normal ranges in broilers given BFRE. Chickens exposed to low ambient temperatures divert part of their production energy to maintain thermal homeostasis. An increase in feed intake and decrease in growth have been reported in association with reduced feed efficiency (Shinder et al., 2002). The manipulation of dietary protein content, energy density and calcium levels as well as the use of vitamins $\mathrm{C}$ and $\mathrm{E}$ are practices believed to alleviate the effects of cold stress in chickens (Sahin et al., 2003). Under stressful environments, physiological requirements for bioactive elements may exceed the synthesizing ability of chickens, which might explain the effectiveness of BFRE.

Furthermore, the activity of marker enzymes which was monitored as CK, LDH-L and AST activity, showed more dependency on the BFRE particularly when exposed to long periods of cold stress. The reduction of plasma CK activity is indicative of down regulated skeletal muscle viability and is a consequence of the reduction in muscle cell membrane function and permeability (Mitchell \& Sandercock, 1995). The finding that serum enzymatic activities were within the normal range reflects a normal metabolism in the broilers fed diets containing BFRE.

Reports suggested that exogenous stimuli would eventually result in oxidative stress in animals (Gumuslu et al., 2002; Teshfam et al., 2006). Many active elements such as flavonoids, phenolic acids, lactones, polysaccharides, amino acids, were identified in bamboo extracts and evaluated in vitro for free radical scavenging and antioxidant activities (Hu et al., 2000). These components might have contributed to the ability of BFRE to alleviate the consequences of cold stress in the present study.

\section{Conclusion}

Cold stress is one of the most challenging environmental conditions that affect commercial poultry in certain countries. BFRE, investigated in this study, could effectively prevent chickens from a reduced body weight gain, and in maintaining serum enzymatic activities during cold stress, and appears to be an effective additive to minimize the effects of cold stress on broilers.

\section{Acknowledgements}

This work was supported by the grant from the China S \& T Pillar Programme (NO. 2008BADC1B01, NO. 2008BADA9B0603), and from Hefei Comprehensive Experimental Station, the National broiler industrial system of China (nycytx-42-Z14).

\section{References}

Cao, H.Q., Yue, Y.D., Peng, Z.H., Hua, R.M. \& Tang, F., 2005. Determination of the antifungal activity of Phyllostachys pubescens extract and raw purification of its antifungal components. Acta Phytopathol. Sin. 35, 428-433.

Castaldo, S. \& Capasso, F., 2002. Propolis, an old remedy used in modern medicine. Fitoterapia 73, S1-S6.

Chuyen, N.V., Kurata, T., Kato, H. \& Fujimaki, M., 1982. Antimicrobial activity of Kumazasa (Sasa albomarginata). Agr. Biol. Chem. 46, 971-978.

Fakeye, T., 2008. Toxicity and immunomodulatory activity of fractions of Hibiscus sabdariffa Linn (family Malvaceae) in animal models. Afr. J. Trad. Comp. Alt. Med. 5, 394-398.

Feng, J., Zhang, M., Zheng, S., Xie, P. \& Ma, A., 2008. Effects of high temperature on multiple parameters of broilers in vitro and in vivo. Poult. Sci. 87, 2133-2139. 
Gumuslu, S., Sarikcioglu, S.B., Sahin, E., Yargicoglu, P. \& Agar, A., 2002. Influences of different stress models on the antioxidant status and lipid peroxidation in rat erythrocytes. Free Radical Res. 36, $1277-1282$.

Hangalapura, B.N., Nieuwland, M.G.B., Buyse, J., Kemp, B. \& Parmentier, H.K., 2004. Effect of duration of cold stress on plasma adrenal and thyroid hormone levels and immune responses in chicken lines divergently selected for antibody responses. Poult. Sci. 83, 1644-1649.

Hu, CH., Zhang, Y. \& David, D.K., 2000. Evaluation of antioxidant and prooxidant activities of bamboo Phyllostachys nigra var. henonis leaf extract in vitro. J. Agric. Food Chem. 48, 3170-3176.

Kumari, P., Gupta, M.K., Ranjan, R., Singh, K.K. \& Yadava, R.Y., 2007. Curcuma longa as feed additive in broiler birds and its patho-physiological effects. Indian J. Exp. Biol. 45, 272-277.

Mekbungwan, A., Yamauchi, K., Sakaida, T. \& Buwjoom, T., 2008. Effects of a charcoal powder-wood vinegar compound solution in piglets for raw pigeon pea seed meal. Animal 2, 366-374.

Mitchell, M.A. \& Sandercock, D.A., 1995. Creatine kinase isoenzyme profiles in the plasma of the domestic fowl (Gallus domesticus): Effects of acute heat stress. Res. Vet. Sci. 59, 30-34.

Mujahid, A., Akiba, Y. \& Toyomizu, M., 2007. Acute heat stress induces oxidative stress and decreases adaptation in young white leghorn cockerels by down regulation of avian uncoupling protein. Poult. Sci. 86, 364-371.

Mujahid, A., Akiba, Y. \& Toyomizu, M., 2009. Olive oil-supplemented diet alleviates acute heat stressinduced mitochondrial ROS production in chicken skeletal muscle. Am. J. Physiol-Reg. I. 297, R690R698.

Sahin, K., Onderci, M., Sahin, N., Ferit Gursu, M. \& Aydin, S., 2003. Cold-induced elevation of homocysteine and lipid peroxidation can be alleviated by dietary folic acid supplementation. Nutr. Res. 23, 357-365.

Samanya, M. \& Yamauchi, K., 2001. Morphological changes of the intestinal villi in chickens fed the dietary charcoal powder including wood vinegar compounds. J. Poult. Sci. 38, 289-301.

SAS, 2001. Statistical Analysis Systems user's guide. SAS Institute Inc., Raleigh, North Carolina, USA.

Shinder, D., Luger, D., Rusal, M., Rzepakovsky, V., Bresler, V. \& Yahav, S., 2002. Early age cold conditioning in broiler chickens (Gallus domesticus): thermotolerance and growth responses. J. Therm. Biol. 27, 517-523.

Shinder, D., Rusal, M., Tanny, J., Druyan, S. \& Yahav, S., 2007. Thermoregulatory responses of chicks (Gallus domesticus) to low ambient temperatures at an early age. Poult. Sci. 86, 2200-2209.

Sun, P.M., Liu, Y.T., Zhao, Y.G., Bao, E.D. \& Wang, Z.L., 2007. Relationship between heat damages and HSPs mRNA in persistent heat stressed broilers. Agric. Sci. China 6, 227-233.

Teshfam, M., Brujeni, G.N. \& Hassanpour, H., 2006. Evaluation of andothelial and inducible nitric oxide synthase mRNA expression in the lung of broiler chickens with developmental pulmonary hypertension due to cold stress. Br. Poult. Sci. 47, 223-229.

Wang, H.X. \& Ng, T.B., 2003. Dendrocin, a distinctive antifungal protein from bamboo shoots. Biochem. Bioph. Res. Co. 307, 750-755.

Yahav, S., 2000. Domestic fowl - strategies to confront environmental conditions. Avian Poult. Biol. Rev. $11,81-95$.

Yan, J.Y., Bao, E.D. \& Yu, J.M., 2009. Heat shock protein 60 expression in heart, liver and kidney of broilers exposed to high temperature. Res. Vet. Sci. 86, 533-538. 